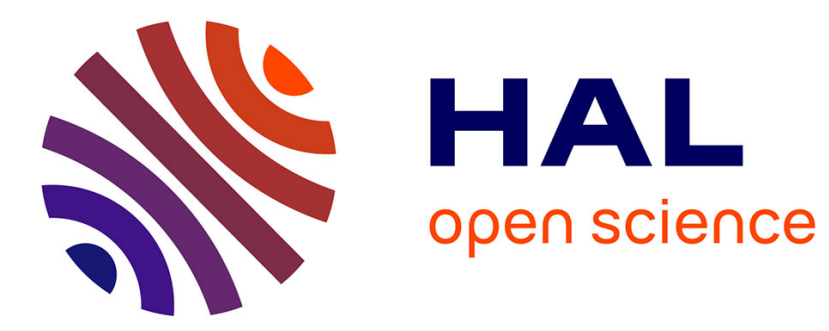

\title{
Résultats de l'expérience à 4 faisceaux en irradiation sphérique à Limeil
}

D. Billon, P.A. Holstein, J. Launspach, C. Patou, D. Schirmann

\section{To cite this version:}

D. Billon, P.A. Holstein, J. Launspach, C. Patou, D. Schirmann. Résultats de l'expérience à 4 faisceaux en irradiation sphérique à Limeil. Revue de Physique Appliquée, 1977, 12 (7), pp.1023-1028. 10.1051/rphysap:019770012070102300 . jpa-00244271

\section{HAL Id: jpa-00244271 https://hal.science/jpa-00244271}

Submitted on 1 Jan 1977

HAL is a multi-disciplinary open access archive for the deposit and dissemination of scientific research documents, whether they are published or not. The documents may come from teaching and research institutions in France or abroad, or from public or private research centers.
L'archive ouverte pluridisciplinaire HAL, est destinée au dépôt et à la diffusion de documents scientifiques de niveau recherche, publiés ou non, émanant des établissements d'enseignement et de recherche français ou étrangers, des laboratoires publics ou privés. 


\title{
RÉSULTATS DE L'EXPÉRIENCE A 4 FAISCEAUX EN IRRADIATION SPHÉRIQUE A LIMEIL (*)
}

\author{
D. BILlON, P. A. HOLSTEIN, J. LAUNSPACH, C. PATOU et D. SCHIRMANN \\ Commissariat à l'Energie Atomique, Centre d'Etudes de Limeil \\ B. P. no 27, 94190 Villeneuve Saint Georges, France
}

(Reçu le 11 janvier 1977, accepté le 24 mars 1977)

\begin{abstract}
Résumé. - Nous rendons compte de résultats obtenus sur l'expérience d'implosion IRIS réalisée à Limeil au moyen des quatre faisceaux émis par le système laser à verre dopé au $\mathrm{NdC}_{6}$ en impulsion nanoseconde et dans une gamme de flux inférieure à $10^{14} \mathrm{~W} / \mathrm{cm}^{2}$. Dans la première partie nous donnerons les résultats d'une étude systématique de l'efficacité d'absorption en fonction des conditions de focalisation des faisceaux laser sur la cible. La seconde partie concernera l'étude spatiotemporelle de l'implosion de microballons en impulsion nanoseconde et à flux laser modérés $\left(<10^{13} \mathrm{~W} / \mathrm{cm}^{2}\right)$.
\end{abstract}

\begin{abstract}
Results obtained on the IRIS implosion experiment are presented. They were obtained using our four beam $\mathrm{Nd}$ glass laser system $\mathrm{C}_{6}$, with a nanosecond pulse, with a flux lower than $10^{14} \mathrm{~W} / \mathrm{cm}^{2}$. Firstly, the results are given of a systematic study of absorption efficiency as a function of laser beam focusing conditions onto the target. Secondly, a spatio-temporal study of microballoon implosion with a nanosecond pulse at a moderate flux $\left(<10^{13} \mathrm{~W} / \mathrm{cm}^{2}\right)$ is presented.
\end{abstract}

1. Introduction. - Nous nous proposons de décrire les résultats expérimentaux obtenus sur l'expérience d'implosion IRIS [1] réalisée à Limeil avec les quatre faisceaux émis par le système laser à verre dopé au Néodyme $\mathrm{C}_{6}$ [2]. Les faisceaux sont focalisés avec des lentilles ouvertes à $f / 2$ sur des cibles sphériques pleines constituées de polyéthylène ou des microballons de verre. Il faut souligner que cette expérience met en jeu une durée relativement longue pour l'impulsion laser ( $>1 \mathrm{~ns}$ ), ce qui permet la mise en place de l'hydrodynamique pendant le dépôt de l'énergie. D'autre part, les valeurs du flux laser absorbé $\left(<10^{14} \mathrm{~W} / \mathrm{cm}^{2}\right)$ sont modérées.

Dans la première partie, nous donnerons les résultats d'une étude systématique de l'optimisation de l'efficacité d'absorption de l'énergie laser en fonction des conditions de focalisation des faisceaux sur la cible [3].

La seconde partie concernera les résultats obtenus lors de l'implosion de microballons de verre à faible rapport d'aspect et en impulsion longue [4].

2. Etude de l'efficacité d'absorption. - 2.1 ConDITIONS EXPÉRIMENTALES. - Les sphères sont éclairées sous vide en géométrie tétraédrique par les 4 faisceaux émis par le laser $\mathrm{C}_{6}$. L'énergie totale $E_{\mathrm{i}}$ incidente, sur la cible est de l'ordre de $140 \mathrm{~J}$. Elle est délivrée sous la

(*) Communication présentée au Congrès National de Physique des Plasmas, Paris, 6-10 décembre 1976. forme d'une impulsion de largeur à mi-hauteur $1,4 \mathrm{~ns}$ et de temps de montée 1,1 ns entre $P_{\mathrm{M}} / 1000$ et $P_{\mathrm{M}}$. La focalisation est assurée au moyen de lentilles asphériques ouvertes à $f / 2$. La structure du faisceau laser et la qualité des lentilles ont été étudiées pour que le diamètre de la tache focale de l'ensemble laser-lentille $(\leqslant 50 \mu \mathrm{m})$ soit toujours petit devant celui des cibles $(100 \mu \mathrm{m}<2 R<300 \mu \mathrm{m})$.

Les points de focalisation des faisceaux sont placés à une même distance $d$ au-delà du centre de la cible.

L'uniformité d'éclairement dépend essentiellement du paramètre $d / R$.

Dans notre cas, si l'on focalise les faisceaux au centre de la cible $(d / R=0)$, seulement $12 \%$ de la surface de celle-ci sont éclairés. Il faut atteindre $d / R=3,6$ pour que la totalité de la surface le soit par au moins un faisceau laser. Quand les rayons lumineux ne sont pas parallèles au gradient de densité qui s'établit autour de la cible, ils subissent une réfraction qui augmente avec l'angle d'incidence et entraîne une diminution de l'absorption.

2.2 CAlCuls NUMÉRIQUES DU TRAJET DES RAYONS LUMINEUX ET DE L'EFFICACITÉ D'ABSORPTION. - Pour nous rendre compte de l'importance de la réfraction nous avons mis au point un code monodimensionnel ABSORBLAS.

Les hypothèses sont les suivantes :

- gradient de densité sphérique, 
- absence d'aberrations géométriques dues aux lentilles et aux faisceaux laser,

- absorption de la lumière uniquement par bremsstrahlung inverse. En particulier, il n'est pas tenu compte de l'absorption résonnante, mécanisme qui, dans nos expériences, reste du second ordre en raison de la valeur relativement faible de la température du plasma dans la zone d'absorption et de la longueur caractéristique élevée du gradient de densité $(\simeq 100 \mu \mathrm{m})[5]$.

La figure 1 représente la variation théorique du pourcentage d'énergie absorbée $\left(E_{\mathrm{a}} / E_{\mathrm{i}}\right)$ en fonction de $d / R$,

\section{$\mathrm{CH}_{2}$}

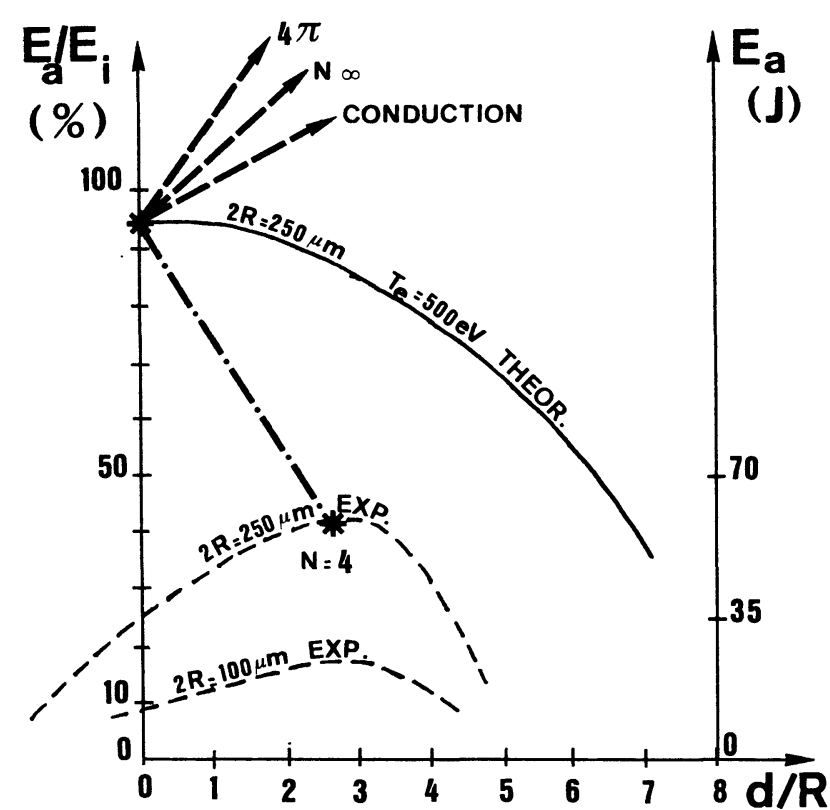

Fig. 1. - Efficacité d'absorption en fonction du rapport de défocalisation. Valeurs théoriques (trait plein) et expérimentales (pointillés).

[Absorption efficiency as a function of defocusing parameter. Theoretical (full line) and experimental (dotted line) values.]

pour une cible en $\mathrm{CH}_{2}$ de diamètre $2 R_{\mathrm{c}}=250 \mu \mathrm{m}$ à la température électronique $T_{\mathrm{e}}=500 \mathrm{eV}$; le gradient de densité provient d'un code [6]. $E_{\mathrm{a}} / E_{\mathrm{i}}$ est maximum pour $d / R=0$; lorsque $d / R$ augmente, l'augmentation de la réfraction s'accompagne d'une diminution de $E_{\mathrm{a}} / E_{\mathrm{i}}$.

2.3 ETUDE EXPÉRIMENTALE DE L'EFFICACITÉ D'ABSORPTION. - Nous avons effectué une étude systématique de $E_{\mathrm{a}} / E_{\mathrm{i}}$ en fonction de $d / R$. Les résultats expérimentaux pour des cibles en $\mathrm{CH}_{2}$ ont été également reportés sur la figure $1 . E_{\mathrm{a}} / E_{\mathrm{i}}$ n'est pas maximum pour $d / R=0$ comme le prévoit le calcul théorique mais pour $d / R=3 . E_{\mathrm{a}} / E_{\mathrm{i}}$ atteint $40 \%$ pour une cible de $250 \mu \mathrm{m}$ de diamètre.
Pour $d / R<3$ et en particulier pour $d / R=0$, la chute de $E_{\mathrm{a}} / E_{\mathrm{i}}$ provient à la fois :

- de la non-uniformité du dépôt d'énergie qui n'est pas lissée par la conduction thermique et qui conduit à une expansion non sphérique de la surface critique entraînant une augmentation des pertes par réfraction,

- et de l'augmentation de la densité de flux donc de $T_{\mathrm{e}}$ entraînant par conséquent une diminution du coefficient d'absorption.

Pour $d / R>3$, la chute de $E_{\mathrm{a}} / E_{\mathrm{i}}$ est due à l'augmentation avec $d / R$ de la réfraction sur le plasma sphérique, et aussi au fait que des rayons passent au-delà du plasma pour $d / R>4$. $d / R=3$ apparaît ainsi comme la valeur minimale de $d / R$ permettant d'obtenir une expansion sphérique.

Pour des cibles de $100 \mu \mathrm{m}$ de diamètre, un taux d'absorption maximal $\left(E_{\mathrm{a}} / E_{\mathrm{i}}\right)_{\max }=18 \%$ est également obtenu pour $d / R=3$, ce qui signifie que, bien que la distance entre les inhomogénéités d'éclairement diminue, la conduction thermique n'est pas suffisante pour symétriser le plasma.

La géométrie de la couronne de plasma a été étudiée par interférométrie à $0,53 \mu \mathrm{m}$, ce qui permet d'explorer la région où $n_{\mathrm{e}}<10^{20} \mathrm{el} / \mathrm{cm}^{3}$ et par photographie de l'émission $\mathrm{X}$ de la zone de conduction électronique. Cette étude a montré une bonne symétrie de la couronne de plasma, pour $d / R \geqslant 3$. Lorsque l'on fait varier le diamètre de la cible (Fig. 2), pour $d / R=3$

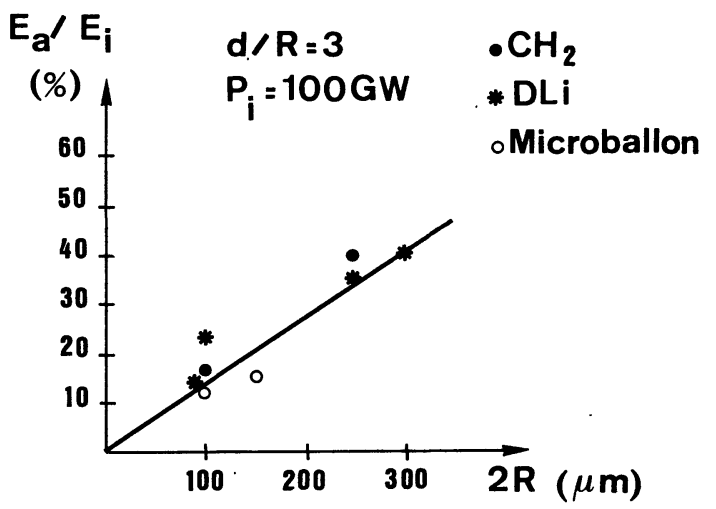

Fig. 2. - Efficacité d'absorption en fonction du diamètre de la cible.

[Absorption efficiency as a function of target diameter.]

et une puissance incidente $P_{\mathrm{i}}=100 \mathrm{GW}, E_{\mathrm{a}} / E_{\mathrm{i}}$ croît linéairement avec le diamètre de la cible.

Cette croissance de $E_{\mathrm{a}} / E_{\mathrm{i}}$ avec $d / R$ provient :

- de l'augmentation du coefficient d'absorption lorsque le flux laser sur la cible diminue,

- de l'allongement du trajet des rayons lumineux dans la zone absorbante dû à la modification du gradient de densité.

Ces expériences ont été faites pour des cibles de natures différentes $\left(\mathrm{CH}_{2}, \mathrm{DLi}\right.$, microballons de verre), 
nous n'avons pas constaté de modification de l'absorption.

Les performances du cœur de la cible dépendent directement de l'énergie absorbée par unité de masse $\left(E_{\mathrm{a}} / m\right), m$ étant la masse initiale de la cible. Pour les cibles pleines $\left(\mathrm{CH}_{2}, \mathrm{DLi}\right)$ l'augmentation de masse (en $R^{3}$ ) conduit à une diminution de $E_{\mathrm{a}} / m$ (en $1 / R^{2}$ ) d'où l'intérêt d'employer de petites cibles. A masse de cible donnée, on constate l'intérêt d'utiliser des microballons plutôt que des sphères pleines pour augmenter le rapport $E_{\mathrm{a}} / m$.

2.4 OPTIMISATION DE L'EFFICACITÉ D'ABSORPTION DANS UNE EXPÉRIENCE D'IMPLOSION (Fig. 1). - D'une manière générale, le maximum d'efficacité d'absorption dans une expérience d'implosion est obtenu en focalisant les faisceaux au centre de la cible et si dans ces conditions l'expansion de la surface critique s'effectue de manière sphérique. Il est possible expérimentalement de s'approcher de cette situation :

- soit en utilisant des systèmes focalisateurs dont l'ouverture totale est égale à $4 \pi$, c'est le cas du système utilisé pour les expériences effectuées par KMS Fusion [7]. Ce mode de focalisation est surtout bien adapté aux expériences à deux faisceaux,

- soit en établissant instantanément un plasma à conductibilité thermique élevée permettant un lissage des défauts d'uniformité d'éclairement. Ceci nécessite un front de montée de l'impulsion très raide permettant d'atteindre rapidement un flux laser suffisant pour que la conduction thermique s'établisse. On peut également utiliser la conduction thermique en employant des cibles avec enrobage à faible densité et une impulsion laser précédée d'une préimpulsion (LASL) [8],

- soit enfin, en utilisant un grand nombre de faisceaux, c'est le cas des expériences effectuées à l'Institut Lebedev en U. R.S.S. avec 9 faisceaux ouverts à $f / 2$ [9]. On peut évidemment chercher à combiner plusieurs de ces effets bénéfiques.

Lorsque la condition $d / R=0$ peut être adoptée, l'efficacité d'absorption est alors limitée uniquement par $T_{\mathrm{e}}$ et le gradient de densité. Ces conclusions sont valables tant que le mécanisme d'absorption par bremsstrahlung inverse reste prépondérant

$$
\varnothing_{\mathrm{a}}<10^{14} \mathrm{~W} / \mathrm{cm}^{2} \text {. }
$$

Si le domaine de flux devient plus élevé il faut prendre en compte d'autres processus tels que l'absorption résonnante par exemple, qui peuvent conduire à des conditions de focalisation différentes si l'on souhaite optimiser l'efficacité d'absorption [5].

3. Implosion de microballons. - Bien que des études expérimentales aient été effectuées sur des implosions de sphères pleines $[10,11]$, nous nous limiterons dans la suite de ce texte à la présentation de résultats concernant l'implosion de microballons de verre.
Pour pouvoir imploser des microballons de verre nous avons dû utiliser les conditions expérimentales suivantes :

- de forts contrastes en puissance et en énergie $\left(10^{6}\right.$ et $\left.10^{4}\right)$ pour ne pas détériorer la cible avant d'atteindre la puissance laser maximum,

- une bonne uniformité de l'éclairement, réalisée lorsque $d / R>3$ et des cibles d'épaisseur très homogène (écarts $<10 \%$ ) pour assurer la symétrie.

De plus, les microballons disponibles ayant un diamètre maximum de $150 \mu \mathrm{m}$ et une épaisseur de $1 \mu \mathrm{m}$, il a été nécessaire, pour ne pas évaporer la totalité de la matière avant la focalisation du choc, de réduire considérablement la puissance laser absorbée. Ceci a été réalisé en défocalisant les faisceaux jusqu'à $d / R=6$ et on a ainsi utilisé une puissance absorbée $P_{\mathrm{a}}$ de $5 \mathrm{GW}$.

Dans ces conditions, nous avons étudié l'évolution de l'implosion des microballons au moyen de l'étude spatio-temporelle de l'émission $X$ et de l'émission sur l'harmonique 2 de la fréquence laser $\left(2 \omega_{0}\right)$.

L'émission à $2 \omega_{0}[12,13]$ apparaît au voisinage de la densité critique où s'effectue l'absorption du rayonnement laser. L'émission $X$ permet de suivre le mouvement de la région chaude et suffisamment dense de la zone de conduction, située entre la surface critique et l'arrière du front de choc.

L'observation de l'émission à $2 \omega_{0}$ est effectuée à l'aide d'une caméra à fente.

La direction d'observation étant perpendiculaire à l'axe de l'une des chaînes laser, l'image de la cible est formée sur la fente d'une caméra à balayage.

Nous donnons (Fig. 3) l'isodensitogramme obtenu

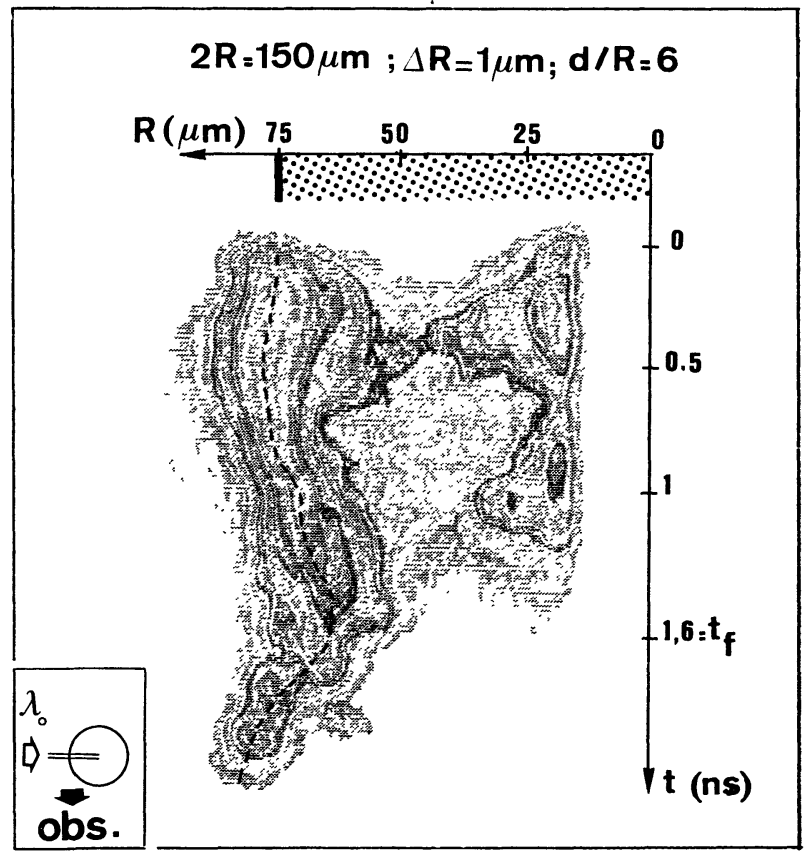

Fig. 3. - Isodensitogramme d'une photographie de l'émission à $2 \omega_{0}$ effectuée à la caméra à fente, à $90^{\circ}$ de l'un des faisceaux. [Isodensitogram of a $2 \omega_{0}$ emission photograph obtained with a streak camera, at $90^{\circ}$ of the direction of a beam.] 
avec une résolution temporelle de 50 ps et une résolution spatiale de $5 \mu \mathrm{m}$. Après une expansion de quelques $\mu \mathrm{m}$, le rayon critique décroît, atteint un minimum égal à $60 \mu \mathrm{m}$ à l'instant $t=1,6 \mathrm{~ns}$ après $P_{\mathrm{M}} / 10$ et croît à nouveau.

La figure 4 montre un isodensitogramme d'une photographie $\mathrm{X}$, intégrée temporellement, obtenue au

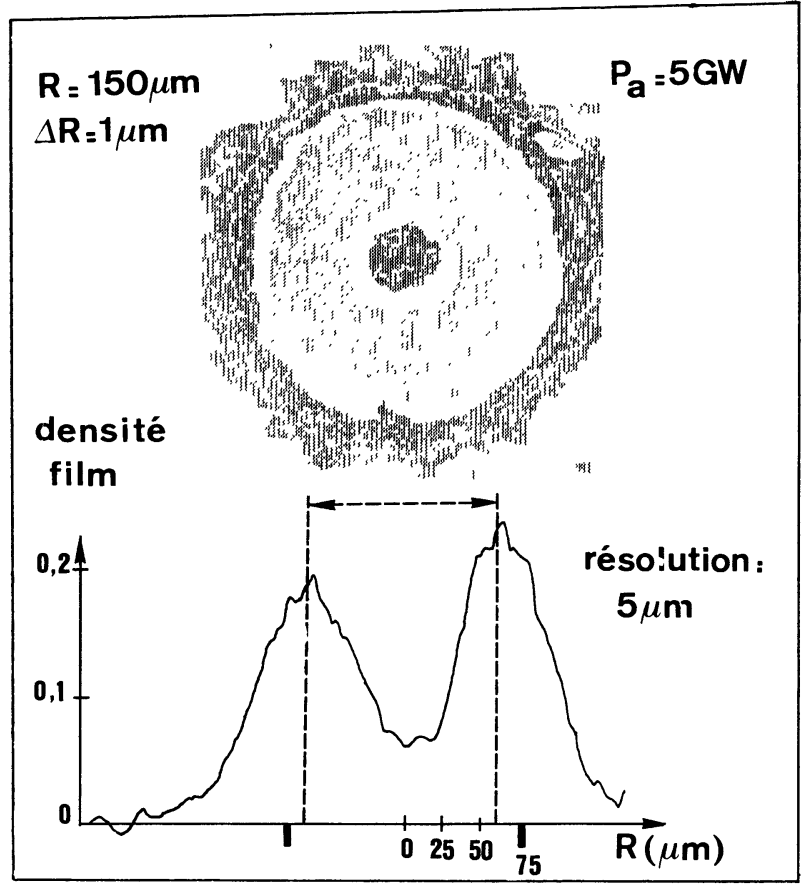

FIG. 4. - Isodensitogramme d'une photographie $\mathrm{X}$ de l'implosion d'un microballon.

[Isodensitogram of the X-ray emission photograph of an imploded microballoon.]

moyen d'une chambre à sténopé. On note une bonne symétrie de la zone de conduction. Le diamètre du maximum d'émission $(125 \mu \mathrm{m})$ est inférieur au diamètre initial de la cible. L'émission $X$ en provenance du cœur est de toute façon insuffisamment contrastée pour être décelable, essentiellement à cause de sa durée $(\simeq 100 \mathrm{ps})$ très inférieure à celle de la couronne ( $\simeq 1,5 \mathrm{~ns})$.

Pour améliorer le rapport signal sur bruit nous avons étudié l'évolution spatio-temporelle de l'émission $X$ au moyen d'une caméra à balayage [14] munie d'une fente placée selon un diamètre de l'image de la cible formée par une chambre à sténopé au grandissement 9,2.

La figure 5 montre les profils d'éclairement sur la fente de la caméra à différents instants durant l'implosion. L'enregistrement a été obtenu derrière $30 \mathrm{mg} / \mathrm{cm}^{2}$ de $\mathrm{Be}$ (atténuation d'un facteur 10 à $2 \mathrm{keV}$ ) avec une résolution spatiale de $20 \mu \mathrm{m}$ et une résolution temporelle de $50 \mathrm{ps}$. La distance entre les maxima de lumination décroît au cours du temps, elle est égale à $120 \mu \mathrm{m}$ à l'instant $t=0,8 \mathrm{~ns}$ après $P_{\mathrm{M}} / 10$; à $t=1,5 \mathrm{~ns}$ elle n'est plus égale qu'à $25 \mu \mathrm{m}$. A l'instant $t=1,6 \mathrm{~ns}$, la résolution spatiale du diagnostic ne permet plus de

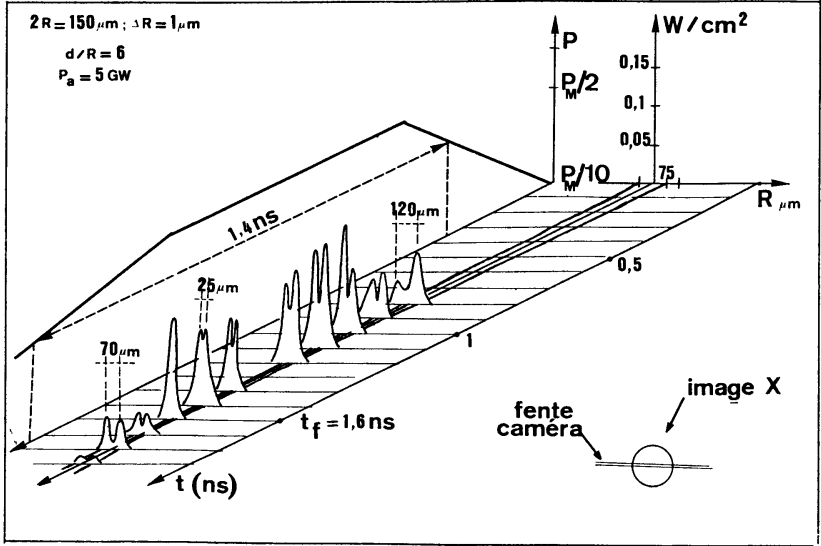

FIG. 5. - Evolution temporelle du profil de lumination déduit d'une photographie $X$ effectuée à la caméra à fente.

[Time evolution of the lumination profile deduced from an X-ray streak photograph.]

résoudre les pics d'émission. A $t>1,6 \mathrm{~ns}$ on observe à nouveau deux maxima de lumination dont l'écart augmente au cours du temps $(70 \mu \mathrm{m}$ à $t=1,8 \mathrm{~ns})$.

Le diamètre de l'émission $X$ et le rayon critique atteignent un minimum au même instant

$$
t=1,6 \mathrm{~ns} \pm 0,1 \mathrm{~ns} .
$$

On verra que l'on est conduit à considérer cet instant comme l'instant de focalisation.

La figure 6 est déduite des profils précédents par intégration le long de la fente de la caméra. A l'instant $t=1,6 \mathrm{~ns}$ on constate une augmentation importante de l'émission $X$ dont la durée est de l'ordre de 100 ps. Cette émission qui apparaît alors que la puissance laser chute peut être attribuée à l'émission $\mathrm{X}$ du cœur au moment de la focalisation. Le cœur est constitué par la fraction de matière non évaporée qui s'accumule au centre, l'accroissement de température provenant de la conversion de son énergie cinétique en énergie thermique. La réduction en volume atteint 240 au moment de la focalisation. La vitesse de l'implosion $\left(5 \times 10^{6} \mathrm{~cm} / \mathrm{s}\right)$ conduit à une température centrale de quelques centaines d'eV.

La figure 7 résume l'ensemble des résultats obtenus sur microballons. Les courbes en pointillé correspondent aux valeurs expérimentales de $R_{\mathrm{c}}$ et du rayon de la zone d'émission $X$. Les courbes en trait plein correspondent aux prévisions numériques effectuées au moyen d'un code hydrodynamique [6] en utilisant l'hypothèse d'un mécanisme d'ablation classique.

On constate un bon accord entre les résultats expérimentaux et théoriques, ce qui tend à prouver que l'évolution de la cible correspond à un mécanisme d'ablation classique. Ceci est justifié par le fait que, compte tenu de la puissance maximale absorbée ( $5 \mathrm{GW})$, le flux laser absorbé est toujours inférieur à $10^{13} \mathrm{~W} / \mathrm{cm}^{2}$.

Ce type d'implosion diffère de celui réalisé à LLL [15] et K. M. S. [16] où sensiblement la même énergie par 


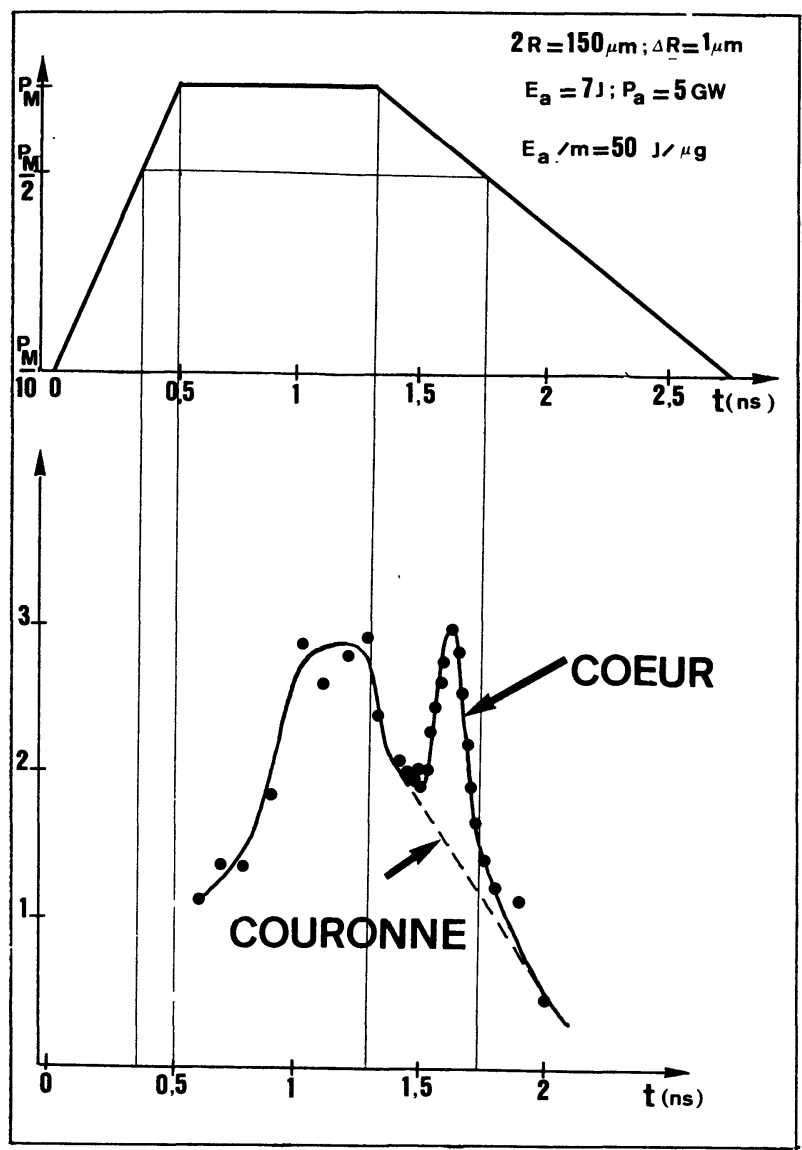

Fig. 6. - Evolution temporelle de l'énergie $X$ intégrée sur la fente de la caméra.

[Time evolution of X-ray energy integrated along the streak camera slide.]

unité de masse, $E_{\mathrm{a}} / \mathrm{m} \simeq 50 \mathrm{~J} / \mu$, est déposée instantanément, donc à très forte puissance, et conduit à une implosion du type exploding pusher. Il est par contre plus proche de celui mis en œuvre dans les expériences poursuivies à l'Institut Lebedev [17].

4. Conclusion. - Une étude systématique de l'efficacité d'absorption en fonction des conditions de focalisation des faisceaux laser sur la cible a montré qu'elle était maximale pour la valeur minimale de $d / R$ permettant d'obtenir une expansion sphérique de la surface critique. Avec 4 faisceaux laser focalisés avec des lentilles ouvertes à $f / 2$ cette valeur est de l'ordre de 3 et entraîne de ce fait des pertes dues à la réfraction des rayons lumineux sur le plasma. Plus généralement l'optimisation de l'efficacité d'absorption dans une expérience d'implosion, où le mécanisme d'absorption par bremsstrahlung inverse reste prépondérant, conduit

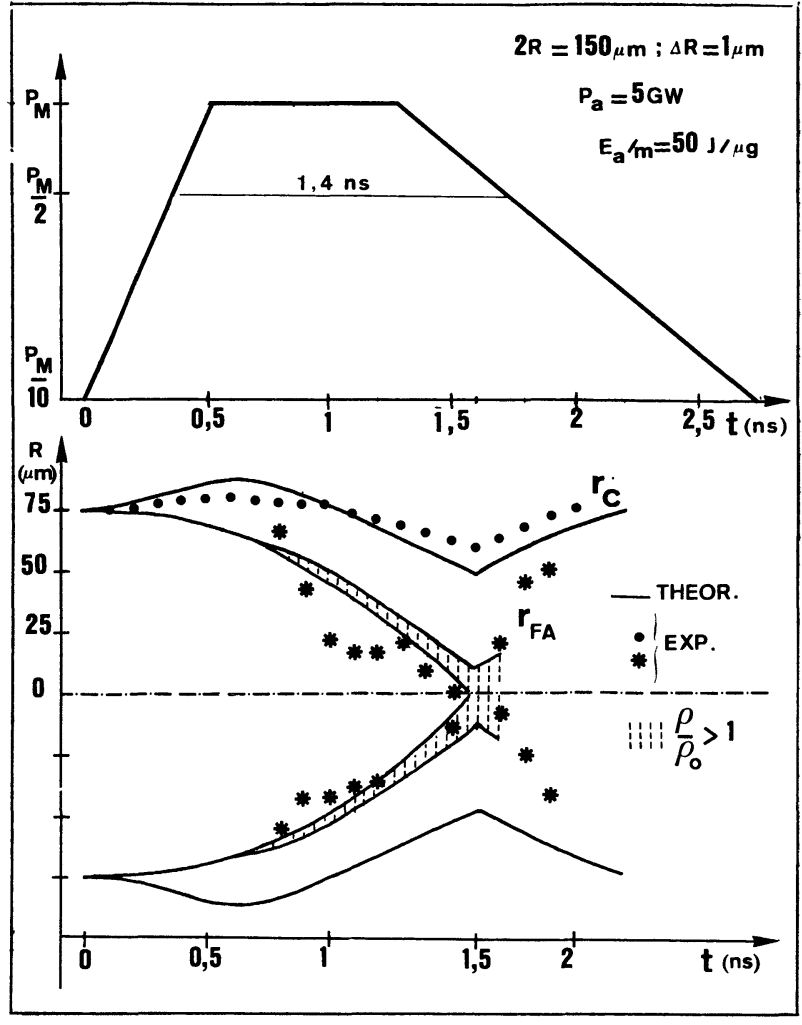

Fig. 7. - Evolution temporelle de la densité critique $\left(r_{\mathrm{c}}\right)$ et du front d'ablation $\left(r_{\mathrm{fa}}\right)$, résultats théoriques et expérimentaux. [Time evolution of critical density $\left(r_{\mathrm{c}}\right)$ and ablation front $\left(r_{\mathrm{FA}}\right)$, theoretical and experimental results.]

à focaliser les faisceaux laser au centre de la cible $(d / R=0)$ avec des conditions d'irradiation assurant en même temps une expansion sphérique de la surface critique. On s'approchera de cette situation idéale en augmentant le nombre des faisceaux laser ou l'ouverture des optiques de focalisation.

A partir de l'émission du plasma sur l'harmonique 2 de la fréquence laser et de l'émission de la zone de conduction dans le domaine $X$, nous avons étudié l'évolution spatio-temporelle de la surface critique et de la zone de conduction dans le cas de l'implosion de microballons en verre de faible rapport d'aspect $(1 / 75)$ réalisée avec des impulsions de durée supérieure à $1 \mathrm{~ns}$. Compte tenu de la gamme de flux utilisée (toujours $<$ à $10^{13} \mathrm{~W} / \mathrm{cm}^{2}$ ) l'évolution de la cible est régie par une ablation classique.

Remerciements. - Les calculs numériques ont été effectués avec le concours de J. M. Reisse et M. Bernard que les auteurs tiennent à remercier particulièrement.

\section{Bibliographie}

[1] Billon, D., Cognard. D., Launspach, J., Patou, C., SchirmanN, D., VIIth Annual Meeting of the APS Division of Plasma Physics, St Petersburg (nov. 1975).
[2] Bardes, J., Bedu, M., Courteille, J. C., Lancelot, J., IIIrd European Electro-Optics Conference (Genève) (oct. 1976). 
[3] Billon, D., Cognard, D., Holstein, P. A., Launspach, J., Patou, C., Schirmann, D., Bernard, M., Reisse, J. M., IXth International Conf. Quant. Electr., Amsterdam (1976).

[4] Billon, D., Holstein, P. A., Launspach, J., Patou, C., SChiRmanN, D., European Conf. on Laser Interaction, Palaiseau (oct. 1976).

[5] Thomson, J. J., Max, C. E., Erkkila, J., Tull, J. E., Phys. Rev. Lett. 37 (1976) 1052.

[6] Bobin, J. L., Reisse, J. M., Revue Phys. Appl. 11 (1976) 497.

[7] Thomas, C. E., Appl. Opt. 14 (1975) 1267.

[8] Mac Call, G. H., MorSe, R. L., Laser Focus (déc. 1974), p. 40.

[9] BASOv, N. G. et al., Laser interaction and related plasma phenomena Volume $3 \mathrm{~B}$, p. 553, édité par H. J. Schwartz et H. Hora (Plenum Press, New York and London) 1974.

[10] Billon, D., Holstein, P. A., Launspach, J., Patou, C., Reisse, J. M., SchirmanN, D., présenté à : IVth Workshop on laser interaction and related phenomena organisé par H. J. Schwartz et H. Hora, Rennselaer Polytechnic Inst., Troy, New York, Nov. 1976.

[11] Launspach, J. et al., présenté à : Congrès de Physique des Plasmas, Parc des Expositions à la porte de Versailles, Paris, 6-10 décembre 1976.

[12] JACKel, S., Perry, B., Lubin, M., Phys. Rev. Lett. 37 (1976) 95.

[13] Saleres, A., Decroisette, M., Patou, C., Opt. Commun. 13 (1975) 321.

[14] Fleurot, N., GeX, J. P., Lamy, M., Quinnesiere, C., SauNEUF, R., XIIth Int. Cong. on High Speed Photography (Toronto) (1976).

[15] Atwood, D. T., Coleman, L. W., European Conf. on Laser Interaction with matter (Palaiseau) (oct. 1976).

[16] Johnson, R. R., Charatis, G. and MAYeR, F. J., KMSF U377, FE-282, OMP-035, 17th Annual Meeting of the division of plasma physics american physical Society, Nov. 10-14, 1975, St Petersburgh, Florida.

[17] Basov, N. G. et al., Lebedev Phys. Institute Ac. Sc. Moscou (1976), Non linear effects in Plasmas, Göteborg (June 1976). 